\title{
Randomized clinical trial on effectiveness of silver diamine fluoride and glass ionomer in arresting dentine caries in preschool children
}

\author{
Qing Hui ZHI ${ }^{\text {a }}$ Edward Chin Man LO ${ }^{\text {b,* }}$, Huan Cai LIN ${ }^{\text {a }}$ \\ ${ }^{a}$ School of Stomatology, Sun Yat-sen University, Guangzhou, China \\ ${ }^{\mathrm{b}}$ Faculty of Dentistry, the University of Hong Kong, Hong Kong SAR,
}

\section{* Correspondence:}

Prof. Edward C.M. Lo

3/F, Prince Philip Dental Hospital, 34 Hospital Road, Hong Kong

E-mail: edward-lo@hku.hk

Tel: 852-2859 0292

Fax: 852-2858 7874

Running title:

SDF and glass ionomer in caries arrest

Key words:

Dental caries, primary dentition, preventive dentistry, fluoride, glass ionomer cement, children

No. of words in abstract: 200

No. of words in text: 2700 (including abstract and acknowledgement)

No. of Figures: 1

No. of Tables: 3

No. of cited references: 23

Based on a thesis submitted to the Faculty of Dentistry, University of Hong Kong, in partial fulfillment of the requirement for a $\mathrm{PhD}$ degree. 


\title{
Randomized clinical trial on effectiveness of silver diamine fluoride and glass ionomer in arresting dentine caries in preschool children
}

\begin{abstract}
Objective: To compare the effectiveness of annual topical application of silver diamine fluoride (SDF) solution, semi-annual topical application of SDF solution, and annual application of a flowable high fluoride-releasing glass ionomer in arresting active dentine caries in primary teeth.
\end{abstract}

Methods: A total of 212 children, aged 3-4 years, were randomly allocated to one of three groups for treatment of carious dentine cavities in their primary teeth: Gp1-annual application of SDF, Gp2-semi-annual application of SDF, and Gp3-annual application of glass ionomer. Follow-up examinations were carried out every six months to assess whether the treated caries lesions had become arrested.

Results: After 24 months, 181 (85\%) children remained in the study. The caries arrest rates were $79 \%, 91 \%$ and $82 \%$ for $\mathrm{Gp} 1, \mathrm{Gp} 2$ and $\mathrm{Gp} 3$, respectively ( $\mathrm{p}=0.007)$. In the logistic regression model using GEE to adjust for clustering effect, higher caries arrest rates were found in lesions treated in $\mathrm{Gp} 2(\mathrm{OR}=2.98, \mathrm{p}=0.007)$, those in anterior teeth $(\mathrm{OR}=5.55, \mathrm{p}<0.001)$, and those in buccal/lingual smooth surfaces $(\mathrm{OR}=15.6, \mathrm{p}=0.004)$. Conclusion: Annual application of either SDF solution or high fluoride-releasing glass ionomer can arrest active dentine caries. Increasing the frequency of application to every 6 months can increase the caries arrest rate of SDF application.

Clinical significance: Arrest of active dentine caries in primary teeth by topical application of SDF solution can be enhanced by increasing the frequency of application from annually to every 6 months, whereas annual paint-on of a flowable glass ionomer can also arrest active dentine caries and may provide a more esthetic outcome. 


\section{INTRODUCTION}

Similar to many developing countries, in China, due to the under-developed oral health care system, most of the decayed teeth in children remain untreated, especially among those living in rural areas where the situation is more serious. ${ }^{1,2}$ Untreated dental caries in children can lead to problems with food intake, sleep, daily activities, and self-esteem. ${ }^{3}$ Thus, simple clinical methods that are effective in treating decayed teeth under field condition are needed for the mass child population.

Traditionally, dental caries is treated by a surgical restorative approach. This requires sophisticated dental equipment and well-trained operators, and is relatively expensive. In recent years, remineralization of caries lesions has gained acceptance in the practice of minimally invasive dentistry and caries arrest treatment is being promoted as part of the basic package of oral care. ${ }^{4,5}$ Among the available methods, topical application of silver diamine fluoride (SDF) solution has been shown to be effective in arresting active caries in primary teeth. ${ }^{6-8}$ In a clinical trial among Chinese preschool children, a 38\% SDF solution was applied once a year onto the caries lesions in primary anterior teeth. ${ }^{6}$ The success rate of SDF in arresting caries after 30 months was around 70\%. In a clinical trial in Cuba, SDF solution was applied onto primary canines and molars, and first permanent molars of school children every 6 months for 36 months. ${ }^{7}$ Most (77\%) of the treated caries lesions that were active at baseline became arrested. Another clinical trial in Nepal found that approximately $35 \%$ of the active carious cavities in primary teeth became arrested at the 24-month follow-up after a single application of $38 \%$ SDF solution. ${ }^{8}$ Whether a higher caries arrest rate can be achieved through more frequent applications is unknown. One of the 
outcomes of SDF applications is that the arrested caries lesion will turn black in color which may not be pleasing. There is a need to look for another effective treatment that can produce a better esthetic.

Glass ionomer has been used as a material for placement of dental restorations and is known to release fluoride which can help to remineralize carious lesion. ${ }^{9}$ Recently, a flowable high fluoride-releasing glass ionomer material is introduced. A laboratory study found that it could release $213 \mu \mathrm{g} / \mathrm{mm}^{2}$ of fluoride in the first day after setting which was double the amount released by a resin-modified glass ionomer. ${ }^{10}$ The high concentration of fluoride ion released may help to arrest active caries and to prevent new dental caries development. Furthermore, its white color makes it a good choice for use in anterior teeth. However, clinical evidence on its effectiveness in arresting dental caries is lacking.

The objective of this clinical trial was to compare the effectiveness of annual topical application of silver diamine fluoride (SDF) solution, semi-annual topical application of SDF solution, and annual application of a flowable high fluoride-releasing glass ionomer in arresting active dentine caries in primary teeth. The null hypothesis to be tested in this clinical trial was that there was no difference in the effectiveness of the three treatments in arresting active caries.

\section{MATERIALS \& METHODS}

The study site was conducted in 2007-2009 in a suburb of Guangzhou, Guangdong 
Province in southern China. Approval from the Independent Review Board of the University of Hong Kong (HK Clinical Trial Register UW07-083) was obtained. The study sample was recruited from the generally healthy children attending six kindergartens. Baseline examinations were conducted by two calibrated examiners in the kindergartens using an intra-oral LED light and disposable mouth-mirrors. After the examination, children who had teeth with active dentine caries not involving the pulp were invited to participate in the trial. Only children with written consent from their parents were accepted. Children who refused dental treatment and teeth that were grossly broken down, more than one third of the crown missing, or pulpally involved were excluded. Tooth with pulp exposure, presence of an abscess or a sinus, obvious discoloration, and premature hypermobility were regarded as pulpally involved tooth.

Status of the dentine caries lesions were assessed by visual inspection and aided by tactile detection using a sharp probe. Cavities with yellowish/brown rough wall/floor which could be easily penetrated by the probe using a light force were diagnosed as active. Those with smooth hard surfaces which could not be penetrated were classified as arrested. Great care was exercised not to damage the teeth when probing was used. Five surfaces in each posterior tooth (occlusal, buccal, lingual, mesial and distal) and four surfaces in each anterior tooth were assessed.

After the examination, children fulfilling the inclusion criteria were allocated individually to one of three treatment groups based on computer-generated random numbers by an assistant. To avoid over clustering, up to 3 decayed teeth in one child, selected using computer-generated random numbers, were included in this study. The 
parents were advised to bring their children to a dentist for treatment of decayed teeth not included in this study.

When providing treatment, the superficial soft decayed tissues of the selected decayed primary tooth were removed by hand instruments. Then, depending on the child’s group allocation, either a 38\% SDF solution (Saforide, Seiyaku Kasei Co. Ltd., Osaka, Japan) or a low viscosity, high fluoride-releasing glass ionomer material (Fuji VII, GC Corporation, Tokyo, Japan) was painted onto the cavity with a small disposable brush. Before application of glass ionomer, the cavity surfaces were conditioned with the conditioner supplied by the manufacturer, washed with a wet cotton pellet, and dried. Children in Group 1 received SDF solution application every 12 months; children in Group 2 received SDF solution application every 6 months; and children in Group 3 received glass ionomer application every 12 months. The children were instructed not to eat or drink for at least 30 minutes after the application.

Follow-up examinations at 6-month intervals were carried out by a single examiner who was not involved in the provision of treatments and did not know the children's group assignment. The treated caries lesions were classified as either active or arrested according to the diagnostic criteria used at baseline. Lesions in Group 3 that were totally covered by the glass ionomer material were classified as arrested. A random $10 \%$ sample of the children were re-examined during each examination to monitor intra-examiner reproducibility.

Questionnaires were completed by the parents at baseline and after 24 months to 
collect information on the child's tooth brushing behavior, use of fluoride toothpaste, frequency of candy or sweet snack intake between meals, and parent's satisfaction with the child's dental appearance.

In the calculation of sample size required, the anticipated 24-month caries arrest rate of annual application of SDF solution was $70 \%{ }^{6}$ An absolute difference of $12 \%$ in rates between groups was regarded as clinically significant. Based on a $5 \%$ statistical significance level and an 80\% power, 200 lesions in each group would be required. Assuming, on average, each child had 4 caries lesions, 50 children were needed in each group. Anticipating a 20\% drop-out rate, 200 children for the three study groups were recruited.

Collected data were entered into computer and analyzed using the software SPSS 16.0 for Windows (SPSS Inc., Chicago, USA) and SAS 9.2 (SAS Institute Inc., Cary, USA). Chi-square test and ANOVA were used, when appropriate, to assess the differences between the three groups of children regarding their demographic background, baseline toothbrushing habit and dmft score, and caries arrest rates at the 24-month evaluation. Since more than one caries lesion were chosen from one child, GEE (Generalized Estimating Equation) approach was used to adjust for the clustering effect and a multi-level non-linear logistic regression model was built. ${ }^{11}$ The dependent variable was whether the lesion was arrested or not at the 24-month examination, and the independent variables included the child's group assignment, gender, toothbrushing frequency, use of fluoride toothpaste, snacking habit, baseline dmft score, tooth type and tooth surface involved. Statistical significance level was set at 0.05 for all tests. 


\section{RESULTS}

At baseline, 481 children were screened and 212 children (114 boys, 98 girls) with a total of 719 active dentine caries lesions meeting the criteria were included and randomly allocated into three treatment groups (Figure 1). There were 71, 69, and 72 children in Groups 1 to 3, respectively.

The mean age of the children was $3.8 \pm 0.6$ years. Most of them did not brush their teeth daily and around two thirds of them used fluoridated toothpaste (Table 1). The mean dmft score of the children at baseline was $5.1 \pm 4.0$. Around half of the included teeth were upper anterior teeth, and the most commonly involved surfaces were mesial, occlusal and distal surfaces. No statistically significant differences were found among the three groups of children in any of the above parameters.

After 24 months, 181 (85\%) children remained in the study, 60, 59 and 62 children in Groups 1 to 3, respectively. The drop-out rates among the three groups were similar $\left(\chi^{2}\right.$ test, $\left.\mathrm{p}>0.05\right)$. There were no statistically significant differences in the baseline toothbrushing habit and mean dmft scores between the children who remained and those who were lost to follow-up (5.0 vs. 5.3; p>0.05).

Intra-examiner reproducibility was good, with Cohen's Kappa statistic values greater than 0.9 in the baseline and follow-up examinations. As shown in Table 2, the caries arrest rates of the treatments found at the 24-month examination were $79 \%$, 91\% and 82\% for Group 1 (SDF 12-month), Group 2 (SDF 6-month), and Group 3 
(GI 12-month), respectively ( $\chi^{2}$ test, $\left.\mathrm{p}=0.007\right)$. Presence of glass ionomer material was found in only 3.5\% (8 out of 229) of the treated lesions in Group 3. Most of the successful cases (172 out of 180) in Group 3 were arrested lesions. No adverse side effects on the treated teeth and soft tissues were found.

Three variables remained in the final multi-level logistic regression model, namely group assignment, tooth type, and tooth surfaces (Table 3). The variables that were not statistically significant were gender (boy vs. girl), toothbrushing frequency (twice daily vs. once daily or less often), frequency of sweet snack intake (twice or more daily vs. once daily or less often), and baseline dmft score. Lesions treated by semi-annual topical application of SDF (Group 2), those in anterior teeth, and those in the buccal or lingual tooth surfaces had a higher chance to become arrested. Compared to those in Group 1, caries lesions in Group 2 had 2.98 times chance to become arrested ( $p=0.007)$ while lesions in Group 3 had a similar chance $(p>0.05)$. Treatments for caries lesions in anterior teeth had 5.55 times chance compared to posterior teeth to arrest the caries $(\mathrm{p}<0.001)$. Caries lesions in buccal or lingual tooth surfaces had 15.6 times chance compared to those on occlusal or approximal surfaces to become arrested $(\mathrm{p}=0.004)$.

Regarding the color of the arrested caries lesions at the 24-month examination, all except one lesion treated with SDF were black while 82\% of those treated by glass ionomer were yellow/brown $\left(x^{2}\right.$ test, $\left.\mathrm{p}<0.001\right)$. Despite this, around $45 \%$ of the parents were satisfied with the appearance of their child's teeth at the 24-month evaluation, and the differences between the three groups were not statistically significant ( $x^{2}$ test, $\left.\mathrm{p}>0.05\right)$. 


\section{DISCUSSION}

This study was a randomized clinical trial using a parallel group design. For ethical reasons, all children in this study received treatment for their decayed teeth. It has been reported that annual application of SDF is an effective treatment to arrest active dentine caries but blackening of the lesions may not be pleasing. ${ }^{12,13}$ The caries arrest rate of annual application of SDF after 24 months in this study is similar to that found in an earlier study on Chinese preschool children. ${ }^{6}$ In this study, when the frequency of SDF application was increased to every 6 months, the proportion of active dentine that had became arrested increased. This is in line with the general recommendation that high risk children should receive more frequent topical fluoride applications. ${ }^{14,15}$ Application of SDF solution every 6 months coincides with the commonly

recommended frequency of recall visits for high risk patients. ${ }^{16,17}$ Better results in managing dental caries in young children can be expected if SDF solution is applied onto the carious lesions every time a child visits a dentist at this recall frequency. This would be an appropriate alternative treatment to the conventional restorative approach in young children who are uncooperative and in places where the supply of dentist is scarce.

This study is probably the first one to investigate the effectiveness of annual paint-on of a flowable glass ionomer to arrest active dentine caries in primary teeth and found that it could be as effective as annual application of SDF solution. Although more time and manipulation in the process of application is needed, application of a flowable glass ionomer provides an alternative treatment when 
application of SDF solution is not accepted due to its taste or blackening of the arrested lesion. Lighter color of the arrested caries lesion makes application of glass ionomer instead of SDF solution an intriguing method, especially for anterior teeth. Despite this, in this study parental satisfaction with their child's dental appearance was similar among the three treatment groups. This may be because the parents were not too concerned about the color of the arrested lesions or that presence of decayed teeth was a greater problem. Since higher cost, more skillful operator and longer treatment time are needed for application of flowable glass ionmer compared to SDF solution, it is more practical to apply glass ionmer in dental clinics or where appearance is of great concern.

Safety of using silver fluoride solution in preschool children had once been doubted and the high fluoride concentration was the main concern. ${ }^{18}$ So far, no severe side effect has been reported. ${ }^{12}$ It has been estimated that each application of 38\% SDF solution only contains $0.2 \mathrm{mg}$ fluoride, ${ }^{19}$ which is far below the probably toxic dosage of $5 \mathrm{mg} / \mathrm{kg}^{20}$ The amount of fluoride released from the glass ionomer material used in this study is around $0.2 \mathrm{mg} / \mathrm{mm}^{2}$ in one day after initial setting. ${ }^{10}$ These low dosages of fluoride are unlikely to have any systemic health effects and the study treatment methods should be very safe for use in preschool children.

Glass ionomer at a high viscosity has been used for placement of restorations in carious cavities in primary teeth in field setting, especially in the atraumatic restorative treatment (ART) approach. ${ }^{21}$ However, the relatively low retention rate of these ART restorations is a problem. ${ }^{22,23}$ Painting on a layer of flowable glass ionomer to cover the carious cavity and allowing the high release of fluoride to arrest the 
lesion may be a more practical treatment when full restoration of original tooth morphology is not needed. In this study, full retention of the applied glass ionomer material was found in only 3.5\% of the treated lesions after 24 months. Many factors can contribute to this low retention rate, such as the low strength of the flowable glass ionomer which was produced primarily for use as a fissure sealant rather than as a restorative material, the lack of retentive cavity form in the carious cavities in primary teeth, and that only the superficial soft carious dental tissues were removed before application of the glass ionomer.

In this study, information on selected possible confounding factors was collected. Clustering effect of having more than one lesion in a child was adjusted. ${ }^{24}$ In the final multi-level logistic regression model, it was found that the child's demographic background, oral health habits, and baseline caries experience did not significantly influence the outcome, i.e. caries arrest. The effect of having more frequent application of SDF was confirmed. It was also found that lesions in the anterior teeth and buccal/lingual surfaces had a higher chance to become arrested. This is probably because these teeth and surfaces are easier to be cleaned by young children. It is also important to find that upper anterior teeth, being commonly involved in early childhood caries, respond well to caries arrest treatment.

This study shows that regular applications of topical fluoride agents can stop the dental caries process even when the lesion has progressed into dentine. Use of simple caries arrest treatment in outreach community health services will be of great significance in the control of the prevalent dental caries problem in many child populations. ${ }^{25}$ This is especially so in places where accessibility to proper dental care 
is limited.

\section{Conclusions}

Based on the results of this study, the null hypothesis is rejected. It is concluded that while the effectiveness of annual application of SDF solution and that of annual application of a flowable high fluoride-releasing glass ionomer in arresting active dentine caries in primary teeth does not differ significantly, increasing the frequency of application of SDF solution to every 6 months can increase the caries arrest rate.

\section{ACKNOWLEDGEMENT}

This study was funded by the University of Hong Kong and the authors had no conflict of interests.

\section{REFERENCES}

1. Qi XQ. Report of the third national oral health survey in China. Beijing: Publishing House of People's Health, 2008.

2. Wong MC, Lo ECM, Schwarz E, Zhang HG. Oral health status and oral health behaviors in Chinese Children. Journal of Dental Research 2001;80:1459-65.

3. Kagihara LE, Niederhauser VP, Stark M. Assessment, management, and prevention of early childhood caries. Journal of the American Academy of Nurse Practitioners 2009;21:1-10.

4. Murdoch-Kinch CA, McLean ME. Minimally invasive dentistry. Journal of the American Dental Association 2003;134:87-95. 
5. Frencken JE, Holmgren CJ, van Palenstein Helderman WH. Basic package of oral care. Nijmegen: College of Dental Sciences, University of Nijmegen, 2008.

6. Chu CH, Lo ECM, Lin HC. Effectiveness of silver diamine fluoride and sodium fluoride varnish in arresting dentin caries in Chinese pre-school children. Journal of Dental Research 2002;81:767-70.

7. Llodra JC, Rodriguez A, Ferrer B, Menardia V, Ramos T, Morato M. Efficacy of silver diamine fluoride for caries reduction in primary teeth and first permanent molars of schoolchildren: 36-month clinical trial. Journal of Dental Research 2005;84:721-4.

8. Yee R, Holmgren C, Mulder J, Lama D, Walker D, van Palenstein Helderman W. Efficacy of silver diamine fluoride for arresting caries treatment. Journal of Dental Research 2009;88:644-7.

9. Hatibovic-Kofman S, Suljak JP, Koch G. Remineralization of natural carious lesions with a glass ionomer cement. Swedish Dental Journal 1997;21:11-7.

10. Bayrak S, Tunc ES, Aksoy A, Ertas E, Guvenc D, Ozer S. Fluoride release and recharge from different materials used as fissure sealants. European Journal of Dentistry 2010;4:245-50.

11. Hardin WJ, Hilbe MJ. Generalized estimating equations. London: Chapman \& Hall, 2003.

12. Chu CH, Lo EC. Promoting caries arrest in children with silver diamine fluoride: a review. Oral Health Preventive Dentistry 2008;6:315-21.

13. Rosenblatt A, Stamford TC, Niederman R. Silver diamine fluoride: a caries "silver-fluoride bullet". Journal of Dental Research 2009;88:116-25.

14. Azarpazhooh A, Main PA. Fluoride varnish in the prevention of dental caries in children and adolescents: a systematic review. Canadian Dental Association 
Journal 2008;74:73-9.

15. Professionally applied topical fluoride: evidence-based clinical recommendations. Journal of the American Dental Association 2006;137:1151-9.

16. Evans RW, Pakdaman A, Dennison PJ, Howe EL. The Caries Management System: an evidence-based preventive strategy for dental practitioners. Application for adults. Australia Dental Journal 2008;53(1):83-92.

17. Beirne P, Clarkson JE, Worthington HV. Recall intervals for oral health in primary care patients. Cochrane Database Systematic Review 2007(4):CD004346.

18. Gotjamanos T. Safety issues related to the use of silver fluoride in paediatric dentistry. Australia Dental Journal 1997;42:166-8.

19. Chu CH. Effectiveness of silver diamine fluoride and sodium fluoride varnish in arresting dentine caries. PhD thesis. Hong Kong: University of Hong Kong, 2004.

20. Whitford GM. Fluoride in dental products: safety considerations. Journal of Dental Research 1987;66:1056-60.

21. van't Hof MA, Frencken JE, van Palenstein Helderman WH, Holmgren CJ. The atraumatic restorative treatment (ART) approach for managing dental caries: a meta-analysis. International Dental Journal 2006;56:345-51.

22. Yu C, Gao XJ, Deng DM, Yip HK, Smales RJ. Survival of glass ionomer restorations placed in primary molars using atraumatic restorative treatment (ART) and conventional cavity preparations: 2-year results. International Dental Journal 2004;54:42-6.

23. Lo ECM, Luo Y, Fan MW, Wei SH. Clinical investigation of two glass-ionomer 
restoratives used with the atraumatic restorative treatment approach in China: two-years results. Caries Research 2001;35:458-63.

24. Wong MC, Lam KF, Lo ECM. Bayesian analysis of clustered interval-censored data. Journal of Dental Research 2005;84:817-21.

24. Petersen PE. The World Oral Health Report 2003: continuous improvement of oral health in the 21st century--the approach of the WHO Global Oral Health Programme. Community Dentistry and Oral Epidemiology 2003;31(Suppl $1): 3-23$. 
Table 1 Background information of the 212 children in the three study groups at baseline.

\begin{tabular}{|c|c|c|c|c|c|}
\hline & \multicolumn{3}{|c|}{ Group } & \multirow[b]{2}{*}{ p-value } \\
\hline & & $\begin{array}{c}\text { (1) SDF-12m } \\
(\mathrm{n}=71)\end{array}$ & $\begin{array}{c}\text { (2) SDF-6m } \\
(n=69)\end{array}$ & $\begin{array}{c}\text { (3) GI-12m } \\
(n=72)\end{array}$ & \\
\hline Age (years) & & $3.8 \pm 0.6$ & $3.8 \pm 0.6$ & $3.8 \pm 0.6$ & 0.669 \\
\hline Mean dmft score & & $4.8 \pm 4.0$ & $4.9 \pm 3.8$ & $5.5 \pm 4.1$ & 0.475 \\
\hline \multirow[t]{2}{*}{ Gender } & male & $51 \%$ & $63 \%$ & $54 \%$ & \\
\hline & female & $49 \%$ & $38 \%$ & $46 \%$ & 0.366 \\
\hline \multirow[t]{4}{*}{ Toothbrushing } & once a day & $26 \%$ & $30 \%$ & $31 \%$ & \\
\hline & twice or more & $16 \%$ & $15 \%$ & $13 \%$ & \\
\hline & occasionally & $49 \%$ & $49 \%$ & $44 \%$ & \\
\hline & never & $10 \%$ & $6 \%$ & $13 \%$ & 0.848 \\
\hline \multirow[t]{2}{*}{ fluoride toothpaste } & yes & $74 \%$ & $65 \%$ & $66 \%$ & \\
\hline & no & $26 \%$ & $35 \%$ & $34 \%$ & 0.842 \\
\hline Tooth included & upper anterior & $51 \%$ & $54 \%$ & $56 \%$ & \\
\hline \multirow[t]{3}{*}{$(n=599)$} & lower anterior & $10 \%$ & $12 \%$ & $10 \%$ & \\
\hline & upper posterior & $7 \%$ & $8 \%$ & $13 \%$ & \\
\hline & lower posterior & $32 \%$ & $26 \%$ & $20 \%$ & 0.076 \\
\hline Tooth surface & occlusal & $19 \%$ & $24 \%$ & $30 \%$ & \\
\hline \multirow[t]{4}{*}{$(n=719)$} & mesial & $49 \%$ & $44 \%$ & $42 \%$ & \\
\hline & distal & $16 \%$ & $21 \%$ & $14 \%$ & \\
\hline & buccal & $12 \%$ & $9 \%$ & $11 \%$ & \\
\hline & lingual & $5 \%$ & $3 \%$ & $3 \%$ & 0.080 \\
\hline
\end{tabular}


Table 2 Caries arrest rates at the follow-up examinations in the three study groups.

\begin{tabular}{lcccc}
\hline & \multicolumn{4}{c}{ Caries arrest rate (\%) } \\
\cline { 2 - 5 } Group & $6 \mathrm{~m}$ & $12 \mathrm{~m}$ & $18 \mathrm{~m}$ & $24 \mathrm{~m}$ \\
\hline (1) SDF-12m & 31.5 & 37.0 & 77.2 & 79.2 \\
(2) SDF-6m & 43.3 & 53.0 & 82.9 & 90.7 \\
(3) GI-12m & 31.3 & 28.6 & 73.1 & 81.8 \\
& $\mathrm{p}=0.012$ & $\mathrm{p}<0.001$ & $\mathrm{p}>0.05$ & $\mathrm{P}=0.007$ \\
\hline
\end{tabular}


Table 3 Logistic regression model of the caries arrest rate at 24-month examination with clustering effect adjustment by GEE.

\begin{tabular}{llccc}
\hline & Explanatory variables & odds ratio & 95\%CI & P-value \\
\hline Group & $(1) \quad$ SDF-12m* & & & \\
& $(2) \quad$ SDF-6m & 2.98 & $1.35-6.69$ & 0.007 \\
\multirow{2}{*}{ Tooth type } & Posterior* & 1.13 & $0.52-2.45$ & $>0.05$ \\
& anterior & & & \\
Surface & occlusal or approximal* & 5.55 & $3.09-9.98$ & $<0.001$ \\
& buccal or lingual & 15.6 & & \\
& & & & 0.004
\end{tabular}

* reference category 


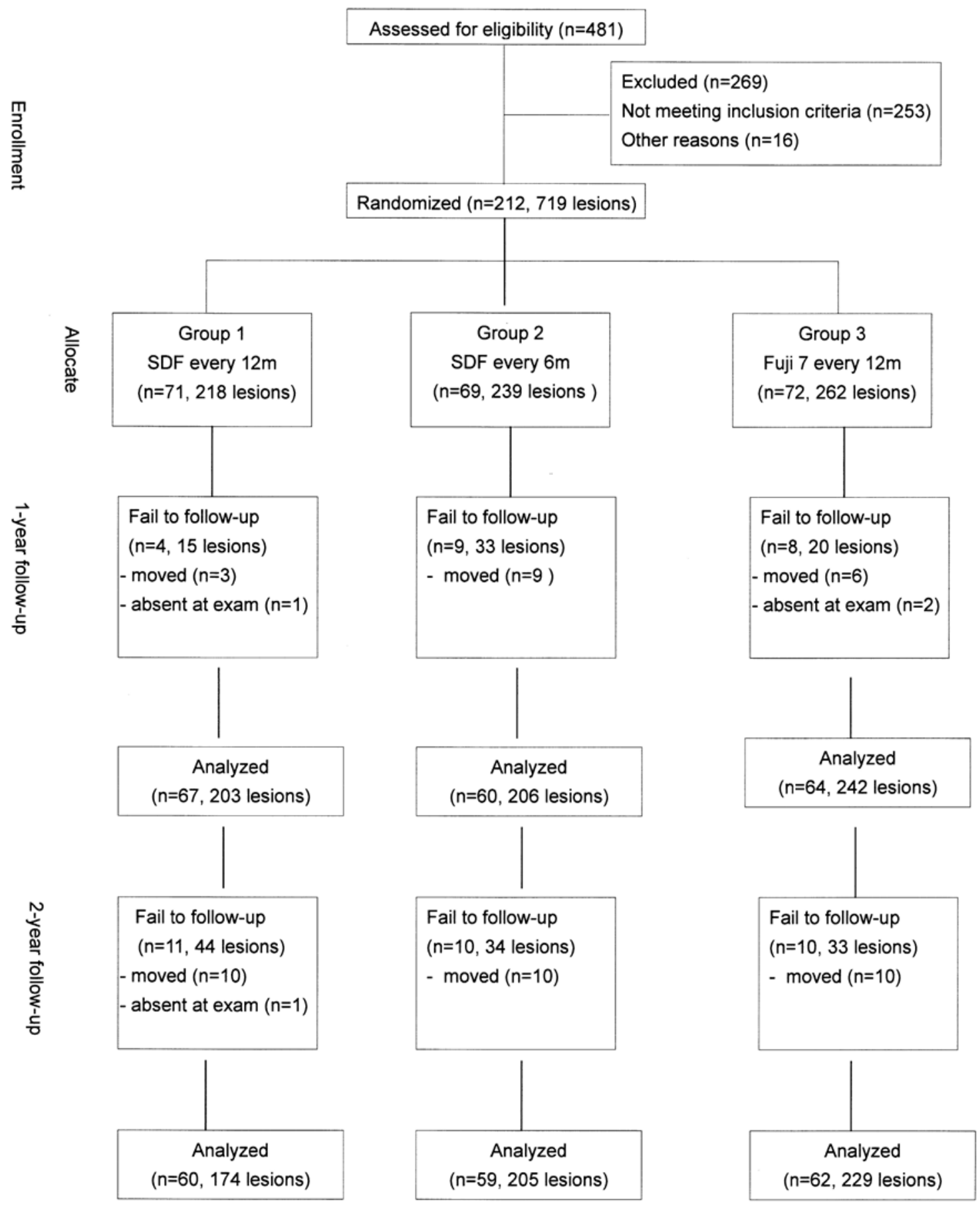

Fig 1 . Flow of subjects over the 24-month study period. 